\title{
Elementi di interfaccia per l'analisi di strutture murarie
}

\author{
Elio Sacco, Jessica Toti \\ Università di Cassino,Di.M.S.A.T., via G.Di Biasio 43, 03043 Cassino (FR), sacco@unicas.it
}

\begin{abstract}
RIASSUNTO. Il presente articolo riguarda la modellazione del comportamento meccanico di elementi in muratura intesi come sistemi eterogenei composti da malta, blocchi ed interfacce di connessione. La strategia computazionale che viene adottata consiste nel modellare separatamente i blocchi, i letti di malta ed le interfacce responsabili di fenomeni di decoesione malta-blocco; a tale scopo, si propone uno speciale modello di interfaccia che combina il danneggiamento con l'attrito. Si sviluppa una procedura numerica, basata sull'algoritmo backward di Eulero, per risolvere il problema evolutivo; per il passo temporale si utilizza invece la tecnica predictor-corrector a controllo di spostamenti. Si effettuano alcune applicazioni numeriche con lo scopo di verificare la capacità del modello e dell'algoritmo proposto nel riprodurre la risposta non lineare della muratura dovuta a fenomeni di degrado localizzati. Infine, si conduce lo studio della modellazione di un arco murario, confrontando i risultati numerici con quelli sperimentali; si dimostra la abilità del modello proposto nel simulare il comportamento globale della struttura ad arco in termini di carico ultimo e di meccanismo di collasso.
\end{abstract}

\begin{abstract}
The present paper deals with the modelling of the mechanical behaviour of masonry elements regarded as heterogeneous systems, made of mortar, bricks and interfaces. Thus, the adopted computational strategy consists in modelling the brick units, the mortar joints and the interfaces responsible for the mortarbrick decohesion mechanisms; to this end, a special interface model combining damage and friction is proposed. A numerical procedure, based on the backward Euler time-integration scheme, is introduced; the time step is solved adopting a displacement driven predictor-corrector scheme. Some numerical applications are performed in order to assess the performances of the proposed model and algorithm in reproducing the nonlinear response of masonry material due to damage localization. Finally, a masonry arch model is studied, comparing the numerical results with experimental ones; it is show the ability of the proposed model to simulate the global behaviour of the arch structure in term of ultimate load and collapse mechanism.
\end{abstract}

Parole Chiave. Interfacce, Danno, Attrito, Muratura, Elementi Finiti.

\section{INTRODUZIONE}

I n molti problemi dell'ingegneria, gli effetti non lineari dei materiali si localizzano in zone di piccolo spessore, dove si sviluppano elevati gradienti di deformazione. Lo spessore di questi strati è così piccolo che spesso viene sostituito nella modellazione da interfacce, che sono particolari superfici di materiale lungo le quali si possono manifestare discontinuità di spostamento.

I modelli di interfaccia sono caratterizzati da relazioni costitutive che legano le tensioni agenti su tali superfici alla discontinuità di spostamento. Molte sono le formulazioni sui comportamenti di interfaccia presenti in letteratura; in particolare, alcune sono state sviluppate per simulare il graduale processo dell'apertura di una fessura, per la quale l'incipiente separazione viene impedita da sforzi coesivi, originati dall'interazione e dall'attrito tra $i$ grani o da altri fenomeni di bridging.

L'idea di adottare un modello coesivo di interfaccia è nata intorno agli anni ' 60 . I primi ad introdurre tale concetto furono Dugdale [1] e Barenblatt [2], che proposero differenti distribuzioni delle tensioni coesive. Successivamente sono state 
proposte altre modellazioni e, per la varietà di problemi di natura numerica e meccanica e per la lunga serie di possibili applicazioni esistenti, sono stati studiati e sviluppati, anche negli ultimi anni, numerosi modelli di zona coesiva.

Relativamente alla modellazione di interfacce in meccanica della muratura, tra gli altri, Lofti and Shing [3] hanno proposto un modello costitutivo di interfaccia capace di simulare l'iniziazione e la propagazione della frattura dovuta all'effetto della presenza delle tensioni normali e tangenziali agenti nella malta e considerando l'effetto della dilatanza. Giambanco e Di Gati [4] hanno formulato un modello coesivo basato su una superficie limite del tipo bilineare alla Coulomb con cut-off a trazione e legge evolutiva non associata. Gambarotta e Lagomarsino [5] hanno sviluppato un modello coesivo di interfaccia che considera l'effetto del danneggiamento e dell'attrito nei giunti di malta soggetti a carichi ciclici. Lourenço e Rots [6] e, successivamente, Oliveira e Lourenço [7] hanno implementato un modello costitutivo di interfaccia basato sulla teoria della plasticità, capace di simulare il comportamento ciclico della zona coesiva, riproducendo la risposta non lineare in fase di scarico.

Giambanco e Mroz [8] hanno presentato un modello di interfase che permette di tenere in conto l'interazione fra le tensioni e le deformazioni di contatto con quelle interne al giunto che risulta separato dai blocchi attraverso due interfacce. Alfano e Sacco [9] hanno proposto un modello di interfaccia che combina il danneggiamento con l'attrito sulla base di una modellazione micromeccanica. Tale modello è stato utilizzato per simulare alcune sperimentazioni; in particolare, è stato riprodotto il comportamento di un pannello murario tramite elementi elastici ed interfacce, che colgono il comportamento sia dei giunti di malta che delle possibili fratture nei blocchi.

Nel presente lavoro si introduce il modello di interfaccia sviluppato da Alfano e Sacco [9], al quale si apportano alcune modifiche. Il modello è capace di simulare il comportamento della connessione malta-blocco. Infatti, la sperimentazione mostra che il fenomeno di distacco della malta dal blocco è il maggiore responsabile della risposta non lineare della connessione tra i blocchi [10]. Il legame costitutivo di interfaccia è determinato sviluppando una semplice ma sistematica analisi micromeccanica del fenomeno della decoesione e dell'attrito. Tale approccio micromeccanico è stato introdotto, nell'ambito della modellazione di un mezzo continuo e coesivo, da Ragueneau et al. [11]. Quindi, l'approccio micromeccanico è stato rivisto da Marfia et al. [12] per sviluppare un modello di interfaccia capace di accoppiare il danneggiamento con l'attrito. Successivamente, è stato ripreso da Uva e Salerno [13] per la modellazione della malta nell'ambito di una procedura di omogeneizzazione di muratura regolare.

Nei paragrafi successivi, si definisce il modello di interfaccia, riportando le equazioni di stato e le leggi evolutive del danno e dell'attrito modellato tramite la teoria della plasticità. Il modello proposto presenta un accoppiamento tra il danneggiamento e l'attrito derivato tramite un semplice ma razionale approccio micromeccanico. Il problema evolutivo non lineare è integrato nel tempo utilizzando un algoritmo backward di Eulero, mentre il passo finito è risolto tramite la tecnica predictor-corrector. Sono quindi illustrate alcune applicazioni numeriche che mostrano la capacità del modello di riprodurre il comportamento dei giunti malta-blocco. Quindi, si mostrano alcune applicazioni su semplici elementi strutturali per verificare la robustezza dell'algoritmo numerico implementato. Infine, viene studiato il comportamento di un arco soggetto a peso proprio ed ad una forza nella prossimità della chiave, confrontando i risultati ottenuti da una sperimentazione sviluppata recentemente [14] con quelli forniti dalla modellazione proposta.

\section{MODELLO COESIVO DI INTERFACCIA}

I n questo paragrafo viene esposto il modello coesivo di interfaccia che accoppia il danno e l'attrito. Tale modello, sviluppato sulla base di quello proposto inizialmente da Alfano e Sacco [9], si basa su un'analisi micromeccanica. Infatti, il legame costitutivo nel generico punto dell'interfaccia, che modella il comportamento della connessione malta-blocco, si ricava considerando l'elemento rappresentativo di area (RAE: Representative Area Element). In Fig. 1 è illustrato schematicamente il modello micromeccanico:

- nel punto A dell'interfaccia malta-blocco, l'elemento rappresentativo RAE della connessione a livello micromeccanico non presenta microfratture e, considerando una schematizzazione semplificata, l'area totale rappresentativa di interazione risulta completamente integra;

- nel punto B, il RAE presenta dei parziali distacchi, ovvero delle microfratture, per cui l'area totale rappresentativa di interazione può essere ripartita in una parte danneggiata ed una integra;

- infine, nel punto $\mathrm{C}$ del giunto di malta, il RAE presenta una frattura completa, per cui l'area totale rappresentativa risulta completamente danneggiata. 


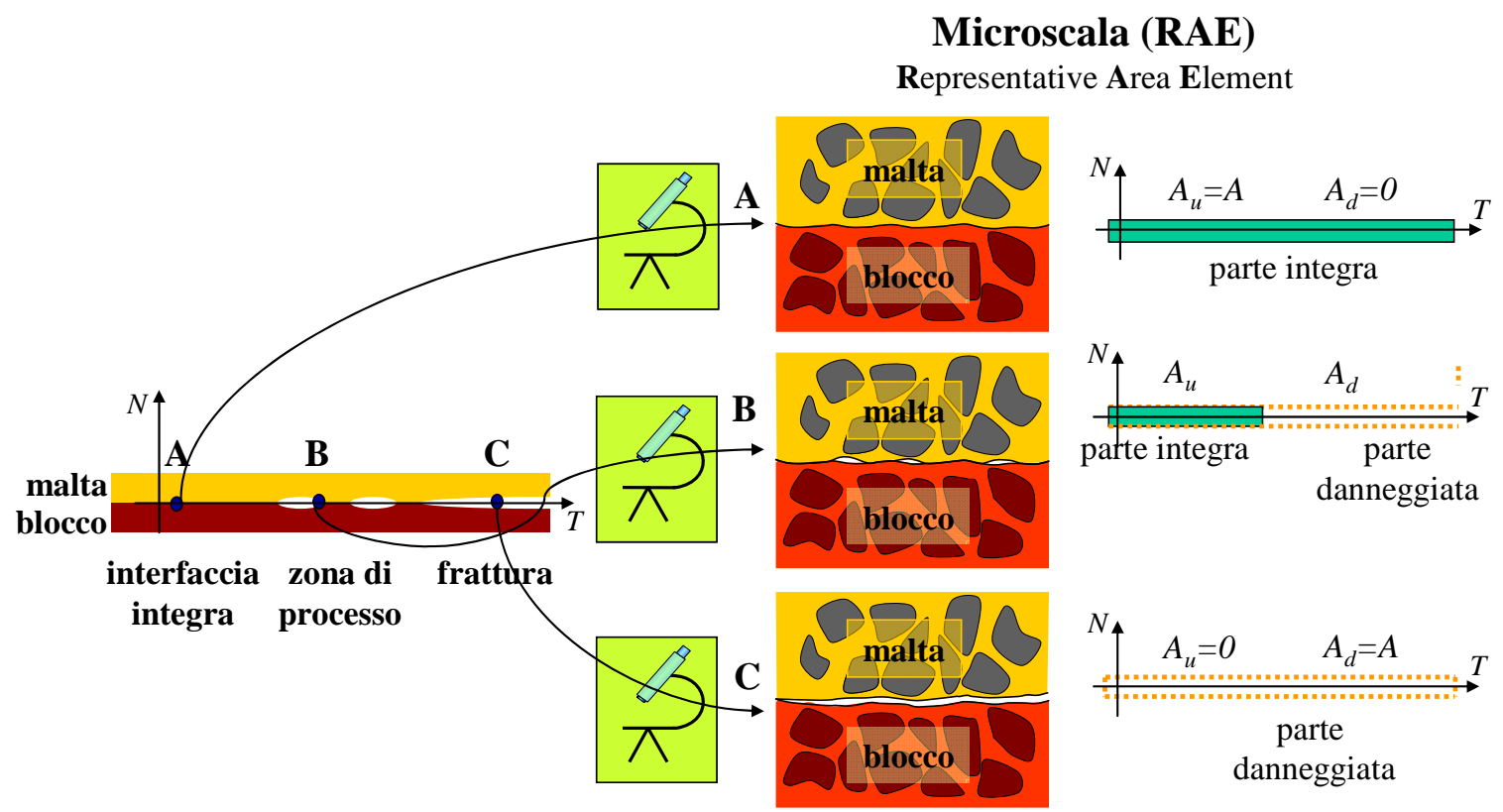

Figura 1: Modello micromeccanico dell'interfaccia malta-blocco.

In definitiva, si assume che l'area rappresentativa elementare del RAE può essere divisa in una parte integra, non danneggiata, $A_{u}$, e una parte completamente danneggiata, $A_{d}$, come illustrato in Fig.1. Il rapporto tra l'area danneggiata e l'area elementare considerata rappresenta il danno e viene denotato con $D$ :

$$
D=\frac{A_{d}}{A}
$$

per cui le due aliquote di area $A_{u}$ e $A_{d}$ posso essere scritte nel seguente modo:

$$
A=A_{d}+A_{u}
$$

con

$A_{u}=(1-D) A, \quad A_{d}=A D$

\section{Cinematica}

Indicando con $\mathbf{s}$ il vettore spostamento relativo in corrispondenza del generico punto dell'interfaccia malta-blocco, si considera il RAE soggetto ad uno spostamento medio pari ad s. Si tratta allora di determinare la distribuzione degli spostamenti relativi che si verificano nel RAE, che rappresenta un punto della zona di interazione malta-blocco. Tale distribuzione di spostamenti relativi dovrebbe essere valutata risolvendo un opportuno modello micromeccanico. Allo scopo di semplificare la trattazione, si può assumere che lo spostamento relativo possa essere considerato costante in ognuna delle due parti di area che compongono il RAE. In particolare, si indica con $\mathbf{s}^{\prime \prime}$ lo spostamento relativo nella parte di area non danneggiata $A_{u}$ e con $\mathbf{s}^{d}$ quello in corrispondenza della zona danneggiata $A_{d}$.

Il vettore spostamento relativo sulla parte danneggiata del RAE risulta diviso in una aliquota elastica $\mathbf{s}^{\text {de }}$ ed una inelastica $\mathbf{s}^{d i}$ :

$$
\mathbf{s}^{d}=\mathbf{s}^{d e}+\mathbf{s}^{d i}
$$

mentre il vettore spostamento relativo, che interessa la zona non danneggiata, è totalmente elastico:

$$
\mathbf{s}^{u}=\mathbf{s}^{\text {ue }}
$$

Gli spostamenti relativi $\mathbf{s}^{\prime \prime}$ e $\mathbf{s}^{d}$, nello spirito dell'analisi micromeccanica, si possono determinare in funzione dello spostamento relativo medio $\mathbf{s}$ in RAE ricorrendo all'uso di opportuni tensori di localizzazione $\mathbf{A}^{d}$ e $\mathbf{A}^{u}$, così che $\mathbf{s}^{d}=\mathbf{A}^{d} \mathbf{s}$ and $\mathbf{s}^{u}=\mathbf{A}^{u} \mathbf{s}$. Un approccio ulteriormente semplificato del problema micromeccanico, che conduce 
comunque ad una formulazione molto soddisfacente relativamente al problema micromeccanico in esame, fu proposto da Voigt [15], assumendo $\mathbf{A}^{d}=\mathbf{A}^{u}=\mathbf{I}$. Quindi, per l'ipotesi appena introdotta, vale l'uguaglianza:

$$
\mathbf{s}^{u}=\mathbf{s}^{d}=\mathbf{s}
$$

Il vettore spostamento relativo ha componenti $\mathbf{s}=\left\{s_{N} s_{T}\right\}^{T}$, avendo indicato rispettivamente con gli indici $N$ e $T$ le componenti nella direzione normale e tangente all'interfaccia, in accordo con il sistema di riferimento locale illustrato in Fig. 1.

\section{Legame costitutivo}

Le tensioni di interfaccia sono differenti sulla parte del RAE danneggiata e su quella non danneggiata. Le tensioni relative all'area danneggiata si denotano con $\tau^{u}$ e sono legate agli spostamenti $\mathbf{s}^{u}$ attraverso un matrice diagonale $\mathbf{K}$ in cui sono contenuti i valori di rigidezza nella direzione normale e tangenziale dell'interfaccia. Considerando l'Eq. (5), si può scrivere la seguente relazione:

$$
\tau^{u}=\mathbf{K} \mathbf{s} \quad \mathbf{K}=\left[\begin{array}{cc}
K_{N} & 0 \\
0 & K_{T}
\end{array}\right]
$$

Sulla parte di area danneggiata le tensioni sono indicate con $\tau^{d}$ e sono legate agli spostamenti elastici di tale zona tramite la seguente espressione :

$$
\tau^{d}=\mathbf{K} \mathbf{s}^{d e}=\mathbf{K}\left[\mathbf{s}-\mathbf{s}^{d i}\right]=\mathbf{K}[\mathbf{s}-(\mathbf{c}+\mathbf{p})]
$$

dove il vettore degli spostamenti anelastici $\quad \mathbf{s}^{d i}=\mathbf{c}+\mathbf{p} \quad$ è decomposto in un vettore $\quad \mathbf{c}=b\left(s_{N}\right)\left\{s_{N} \quad s_{T}\right\}^{T}$ che tiene conto del contatto unilatero e in un vettore

$$
\mathbf{p}=\left\{\begin{array}{ll}
0 & p_{T}
\end{array}\right\}^{T}
$$

che tiene conto dell'effetto di attrito; con $h(\bullet)$ si denota la funzione di Heaviside, pari a

$h(x)=1$ se $x \geq 0$,

$h(x)=0$ se $x<0$

Il valore di tensione dell'interfaccia sull'area elementare rappresentativa viene indicato con $\tau$ e si ottiene come somma pesata dei due valori $\tau^{d}$ e $\tau^{u}$ come segue:

$$
\begin{aligned}
\tau & =(1-D) \tau^{u}+D \tau^{d} \\
& =(1-D) \mathbf{K} \mathbf{s}+D \mathbf{K}[\mathbf{s}-(\mathbf{c}+\mathbf{p})] \\
& =\mathbf{K}[\mathbf{s}-D(\mathbf{c}+\mathbf{p})]
\end{aligned}
$$

Le componenti di tensione normali e tangenziali dei vettori $\tau, \tau^{d}$ e $\tau^{u}$ vengono indicate, rispettivamente, con:

$$
\tau=\left\{\begin{array}{c}
\tau_{N} \\
\tau_{T}
\end{array}\right\}, \quad \tau^{u}=\left\{\begin{array}{c}
\tau_{N}^{u} \\
\tau_{T}^{u}
\end{array}\right\}, \quad \tau^{d}=\left\{\begin{array}{c}
\tau_{N}^{d} \\
\tau_{T}^{d}
\end{array}\right\}
$$

\section{Leggi evolutive}

Lo spostamento relativo inelastico p, che fisicamente rappresenta lo slittamento che avviene sulla zona danneggiata del RAE, è governato dalla classica legge di attrito di Coulomb: 


$$
\phi\left(\tau^{d}\right)=\mu\left\langle\tau_{N}{ }^{d}\right\rangle_{-}+\left|\tau_{T}^{d}\right|=\mu \tau_{N}{ }^{d}+\left|\tau_{T}{ }^{d}\right|
$$

dove $\mu$ rappresenta il coefficiente di attrito e il simbolo $\langle\bullet\rangle_{-}$denota la parte negativa della tensione inelastica. Per l'evoluzione di $\mathbf{p}$ si considera la legge non associata:

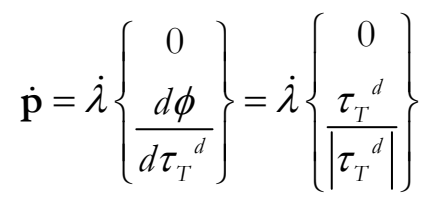

insieme alle condizioni di Khun-Tucker:

$$
\dot{\lambda} \geq 0, \quad \phi\left(\tau^{d}\right) \leq 0, \quad \dot{\lambda} \phi\left(\tau^{d}\right)=0
$$

Relativamente all'evoluzione del parametro di danno $D$ si considera un modello che tiene conto dell'accoppiamento della frattura del modo I e del modo II. Vengono infatti definite due quantità $\eta_{N}$ e $\eta_{T}$ che dipendono dalle tensioni di picco $\tau_{N}^{0}$ e $\tau_{T}^{0}$, dagli spostamenti relativi di prima fessurazione $s_{0 N}$ e $s_{0 T}$ e dalle energie specifiche di frattura $G_{c N}$ e $G_{c T}$ :

$$
\eta_{N}=\frac{s_{0 N} \tau_{N}^{0}}{2 G_{c N}}, \quad \eta_{T}=\frac{s_{0 T} \tau_{T}^{0}}{2 G_{c T}}
$$

Si definisce un parametro $\eta$ che lega i due modi di apertura di frattura:

$$
\eta=1-\frac{1}{\alpha^{2}}\left(\left\langle s_{N}\right\rangle_{+}^{2} \eta_{N}+s_{T}^{2} \eta_{T}\right)
$$

con \langle\rangle$_{+}$parte positiva dell'argomento e $\alpha$ che dipende dalle componenti di spostamento relativo:

$$
\alpha=\sqrt{\left\langle s_{N}\right\rangle_{+}^{2}+\left(s_{T}\right)^{2}}
$$

Il parametro di danno è valutato quindi attraverso la seguente relazione:

$$
D=\max _{\text {bistory }}\langle\min \{1, \tilde{D}\}\rangle
$$

dove:

$$
\tilde{D}=\frac{1}{\eta}\left(\frac{\beta}{1+\beta}\right)
$$

essendo

$$
\beta=\sqrt{\left(\frac{\left\langle s_{N}\right\rangle_{+}}{s_{0 N}}\right)^{2}+\left(\frac{s_{T}}{s_{0 T}}\right)^{2}}-1
$$

\section{Procedura Numerica}

i espone la procedura numerica adottata per la valutazione della risposta meccanica del modello di interfaccia caratterizzato dalle relazioni costitutive precedentemente introdotte. Le equazioni evolutive sono integrate nel tempo adottando una procedura del tipo backward di Eulero con controllo degli spostamenti relativi. 
Il processo di analisi viene infatti diviso in un numero finito di passi; all'istante $t_{n}$ si assume nota la soluzione, le quantità valutate al tempo $t_{n}$ sono contraddistinte dal pedice $n$, mentre le quantità al tempo attuale $t_{n+1}$ non presentano alcun indice. Lo schema di integrazione adottato è il seguente:

- si assume noto lo spostamento relativo di interfaccia $\mathbf{s}$;

- si determina il valore del parametro di danno $D$ tramite le formule (14)-(18);

- si valuta la tensione di prova della zona danneggiata attraverso l'espressione:

$$
\tau_{e l}^{d}=\mathbf{K}\left[\mathbf{s}-\left(\mathbf{c}+\mathbf{p}_{n}\right)\right]
$$

- si determina il valore della funzione limite $\phi\left(\tau_{e l}^{d}\right)$ utilizzando la formula (10).

- se $\phi\left(\tau_{e l}^{d}\right)<0, \mathbf{p}=\mathbf{p}_{n}$ e $\tau^{d}=\tau_{e l}^{d}$

- se $\phi\left(\tau_{e l}^{d}\right) \geq 0$ lo spostamento inelastico deve essere aggiornato tramite le seguenti relazioni:

$$
\mathbf{p}=\mathbf{p}_{n}+\Delta \lambda\left\{\begin{array}{c}
0 \\
\frac{\tau_{T e l}^{d}}{\left|\tau_{T e l}^{d}\right|}
\end{array}\right\} \quad \Delta \lambda=\frac{1}{K_{T}}\left(\mu \tau_{N e l}^{d}+\left|\tau_{T e l}^{d}\right|\right)
$$

- si determina la tensione all'interfaccia tramite l’Eq. (8).

La procedura di integrazione descritta è implementata in un elemento finito interfaccia a quattro nodi, con due gradi di libertà per nodo, e spessore nullo.

In definitiva, nel generico passo temporale finito, si tratta di risolvere il sistema algebrico non lineare scritto in forma residuale:

$$
\begin{aligned}
& \tilde{\mathbf{R}}=\tilde{\boldsymbol{\Lambda}}(\tilde{\mathbf{U}} ; \hat{\mathbf{U}})-\tilde{\mathbf{F}}=\mathbf{0} \\
& \hat{\mathbf{R}}=\hat{\Lambda}(\tilde{\mathbf{U}} ; \hat{\mathbf{U}})-\hat{\mathbf{F}}=\mathbf{0}
\end{aligned}
$$

dove i vettori $\tilde{\mathbf{U}}$ e $\hat{\mathbf{U}}$ rappresentano gli spostamenti nodali liberi incogniti e vincolati, rispettivamente, mentre $\tilde{\mathbf{F}}$ e $\hat{\mathbf{F}}$ sono le forze esterne assegnate e le reazioni vincolari incognite. Il problema algebrico non lineare è risolto sviluppando una procedura iterativa di tipo Newton-Raphson; alla k-esima iterazione, la soluzione si determina tramite le relazioni:

$$
\begin{aligned}
\Delta \tilde{\mathbf{U}}^{k+1} & =-\left(\mathbf{K}_{\tilde{\mathbf{U}} \tilde{\mathbf{U}}}^{t, k}\right)^{-1} \tilde{\mathbf{R}}^{k} \\
\Delta \hat{\mathbf{F}}^{k+1} & =-\hat{\mathbf{R}}^{k}-\mathbf{K}_{\tilde{\mathbf{U}}}^{t, k} \Delta \tilde{\mathbf{U}}^{k+1}
\end{aligned}
$$

dove

$$
\mathbf{K}_{\tilde{\mathbf{U}} \tilde{\mathbf{U}}}^{t, k}=\left.\frac{\partial \tilde{\mathbf{R}}}{\partial \tilde{\mathbf{U}}}\right|_{k} \quad \mathbf{K}_{\hat{\mathbf{U}} \tilde{\mathbf{U}}}^{t, k}=\left.\frac{\partial \hat{\mathbf{R}}}{\partial \tilde{\mathbf{U}}}\right|_{k}
$$

La successione converge quando i residui $\tilde{\mathbf{R}}^{k}$ e $\hat{\mathbf{R}}^{k}$ tendono a zero. Si definisce errore alla k-esima iterazione, la quantità scalare:

$$
e^{k}=\frac{\left\|\mathbf{R}^{k}\right\|}{\left\|\mathbf{R}^{1}\right\|}
$$

La soluzione viene considerata soddisfacente quando accade che l'errore è più piccolo di una prefissata tolleranza, $e^{k}<$ tol .

Il calcolo delle matrici tangenti $\mathbf{K}_{\tilde{U} \tilde{U}}^{t, k}$ e $\mathbf{K}_{\tilde{U} \tilde{U}}^{t, k}$ richiedono la determinazione della derivata consistente con l'algoritmo di integrazione nel tempo del legame $\tau-\mathbf{s}$. Si tratta allora di determinare la derivata $\partial \tau / \partial \mathbf{s}$. Tenuto conto dell'Eq. (8) si ha: 


$$
\mathbf{K}^{t}=\frac{\partial \tau}{\partial \mathbf{s}}=\frac{\partial}{\partial \mathbf{s}}\left[\mathbf{K}(\mathbf{s}-D(\mathbf{c}+\mathbf{p})]=\mathbf{K}\left[\mathbf{I}-(\mathbf{c}+\mathbf{p}) \otimes \frac{\partial D}{\partial \mathbf{s}}-D\left(\frac{\partial \mathbf{c}}{\partial \mathbf{s}}+\frac{\partial \mathbf{p}}{\partial \mathbf{s}}\right)\right]\right.
$$

La derivata del temine $\partial D / \partial \mathbf{s}$ è nulla quando non c'è evoluzione del danno, cioè quando $D=D_{n}$ ovvero $D=1$; tenuto conto della formula (17), si ha:

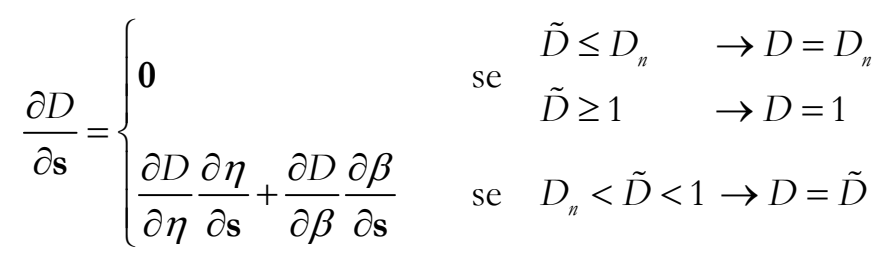

dove, tenendo conto delle Eq. (14)-(18), si ha:

$$
\begin{array}{ll}
\frac{\partial D}{\partial \eta}=-\frac{\beta}{\eta^{2}(1+\beta)} & \frac{\partial D}{\partial \beta}=\frac{1}{\eta(1+\beta)^{2}} \\
\frac{\partial \eta}{\partial \mathbf{s}}=\frac{2}{\alpha^{4}}\left(\eta_{N}-\eta_{T}\right) \tilde{\mathbf{Q s}} & \frac{\partial \beta}{\partial \mathbf{s}}=\frac{1}{1+\beta} \mathbf{A \tilde { s }}
\end{array}
$$

essendo

$$
\mathbf{Q}=\left[\begin{array}{cc}
-s_{T}^{2} & 0 \\
0 & \left\langle s_{N}\right\rangle_{+}^{2}
\end{array}\right] \quad \mathbf{A}=\left[\begin{array}{cc}
\frac{1}{s_{0 N}^{2}} & 0 \\
0 & \frac{1}{s_{0 T}^{2}}
\end{array}\right] \quad \tilde{\mathbf{s}}=\left\{\begin{array}{c}
\left\langle s_{N}\right\rangle \\
s_{T}
\end{array}\right\}
$$

Sostituendo le espressioni delle derivate parziali si ottiene:

$$
\frac{\partial D}{\partial \mathbf{s}}=\frac{1}{\alpha^{4} \eta^{3}(1+\beta)^{3}}\left[\alpha^{4} \eta^{2} \mathbf{A}-2 \beta(1+\beta)^{2}\left(\eta_{N}-\eta_{T}\right) \mathbf{Q}\right] \tilde{\mathbf{s}}=\mathbf{P} \tilde{\mathbf{s}}
$$

La derivata del temine $\partial \mathbf{c} / \partial \mathbf{s}$ è diversa da zero solo quando la componente $s_{N}$ del vettore di spostamento relativo è positivo, in esplicito si ha:

$$
\partial \mathbf{c} / \partial \mathbf{s}=\left\{\begin{array}{lll}
\mathbf{0} & \text { se } & s_{N} \leq 0 \\
\mathbf{I} & \text { se } & s_{N}>0
\end{array}\right.
$$

Per quanto riguarda il termine $\partial \mathbf{p} / \partial \mathbf{s}$, si può verificare il caso in cui nel passo finito non avviene evoluzione dello spostamento relativo inelastico, $\Delta \lambda=0$, oppure c'è evoluzione dello slittamento dovuto all'attrito, $\Delta \lambda>0$. Nel primo caso la derivata è nulla, mentre nel secondo è diversa da zero solo se la componente di spostamento relativo $s_{N}$ assume valore non positivo. In definitiva si ha:

$$
\partial \mathbf{p} / \partial \mathbf{s}=\left\{\begin{array}{lll}
0 & 0 \\
\frac{1}{K_{T}} \mu K_{N} \frac{\tau_{T e l}^{d}}{\left|\tau_{T e l}^{d d}\right|} & 1
\end{array}\right] \quad s_{N} \leq 0 \quad \text { e } \quad \phi\left(\tau^{d}\right) \geq 0
$$




\section{APPLICAZIONI NUMERICHE}

I

1 modello di interfaccia e la procedura numerica proposta nei paragrafi precedenti sono stati utilizzati per sviluppare alcune applicazioni numeriche. Si tratta di tre tipologie di applicazione.

Nella prima parte si riportano i risultati di alcuni dei calcoli effettuati, che hanno avuto lo scopo di verificare le capacità del modello a riprodurre il comportamento delle interfacce coesive. Nella seconda parte si sviluppano alcune analisi di semplici elementi strutturali per verificare la correttezza e le capacità di convergenza della procedura numerica implementata. Nella terza parte viene invece presentata l'applicazione riguardante la modellazione agli elementi finiti di un arco in muratura, la cui risposta in termini di curve forza-spostamento, ottenuta numericamente, viene confrontata con dei risultati sperimentali derivati da una campagna di indagine effettuata precedentemente presso il Laboratorio di Analisi e progettazione Strutturale dell'Università di Cassino, discussi in dettaglio in [14].

\section{Comportamento dellinterfaccia}

Si riportano in tale paragrafo alcuni esempi di risposta meccanica del modello di interfaccia al fine di evidenziarne alcuni aspetti peculiari.

I parametri di interfaccia considerati nell'analisi sono riportati in Tab.1.

\begin{tabular}{|c|c|c|c|c|c|c|}
\hline $\begin{array}{c}\tau_{N}^{0} \\
{\left[\mathrm{~N} / \mathrm{mm}^{2}\right]}\end{array}$ & $\begin{array}{c}G_{c N} \\
{[\mathrm{~N} / \mathrm{mm}]}\end{array}$ & $\begin{array}{c}K_{N} \\
{\left[\mathrm{~N} / \mathrm{mm}^{3}\right]}\end{array}$ & $\begin{array}{c}\tau_{T}^{0} \\
{\left[\mathrm{~N} / \mathrm{mm}^{2}\right]}\end{array}$ & $\begin{array}{c}G_{c T} \\
{[\mathrm{~N} / \mathrm{mm}]}\end{array}$ & $\begin{array}{c}K_{T} \\
{\left[\mathrm{~N} / \mathrm{mm}^{3}\right]}\end{array}$ & $\mu$ \\
\hline 3 & 0.3 & 150 & 3 & 0.3 & 150 & 0.5 \\
\hline
\end{tabular}

Una caratteristica importante del legame di interfaccia introdotto è il comportamento attritivo che viene mostrato attraverso la determinazione delle diverse curve $\tau_{T}-s_{T}$, ottenute incrementando il valore della componente di spostamento relativo tangenziale $s_{T}$ ed assegnando e mantenendo costante durante la storia di carico quella normale $s_{N}$. Come si può notare dalla Fig. 2, la risposta meccanica è caratterizzata dal raggiungimento di una maggiore resistenza tangenziale di picco per i processi di carico contraddistinti da un valore di spostamento normale negativo più elevato in valore assoluto. Inoltre, per le curve caratterizzate da $s_{N} \leq 0$, si ha un comportamento lineare fino al raggiungimento di $\tau_{N}^{0}$, quindi si ottiene un andamento crescente non lineare fino al raggiungimento di una resistenza di picco $\tau_{\max }$. Infine, ad un aumento ulteriore dello spostamento $s_{T}$ corrisponde un decremento di tensione tangenziale, fino al raggiungimento del valore $\tau_{T}=-\mu \tau_{N}$, in corrispondenza del quale il parametro di danno risulta pari ad $1 \mathrm{e}$ la resistenza tangenziale, che rimane costante con $s_{N}$, è solo dovuta all'effetto dell'attrito.

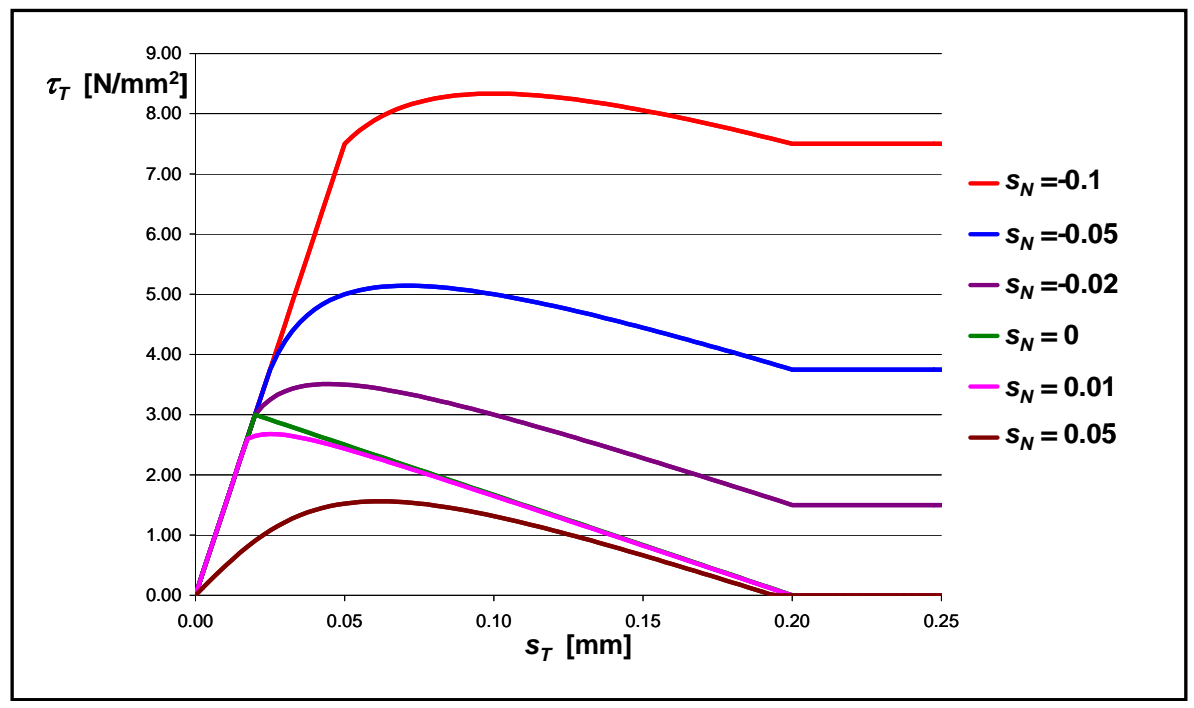

Figura 2: Curve $\tau_{T}-s_{T}$ per differenti valori di $s_{N}$. 
Nel caso in cui, al contrario, è assegnato e mantenuto costante durante tutta la storia di carico uno spostamento normale positivo $\left(s_{N}>0\right)$, la resistenza di picco $\tau_{\max }$ non raggiunge mai $\tau_{N}^{0}$; inoltre, nel caso in cui si ha $s_{N}>s_{0 N}$, anche nel tratto iniziale si può avere un forte comportamento non lineare, perché già all'inizio dell'analisi si ha danneggiamento per effetto della decoesione.

Un altro caso di analisi molto interessante è la risposta meccanica di interfaccia a seguito di una storia di carico ciclica, in cui la componente di spostamento $S_{T}$ assume ai vari tempi i valori riportati in Tab.2, mentre la componente lungo la direzione normale viene invece mantenuta costante.

\begin{tabular}{ccc}
\hline $\begin{array}{c}\text { Tempo } \\
{[\mathrm{s}]}\end{array}$ & $\begin{array}{c}\boldsymbol{s}_{N} \\
{[\mathrm{~mm}]}\end{array}$ & $\begin{array}{c}s_{T} \\
{[\mathrm{~mm}]}\end{array}$ \\
0 & -0.07 & 0 \\
1 & -0.07 & 0.08 \\
2 & -0.07 & -0.10 \\
3 & -0.07 & 0.30 \\
\hline
\end{tabular}

Tabella 2: Storia di carico.

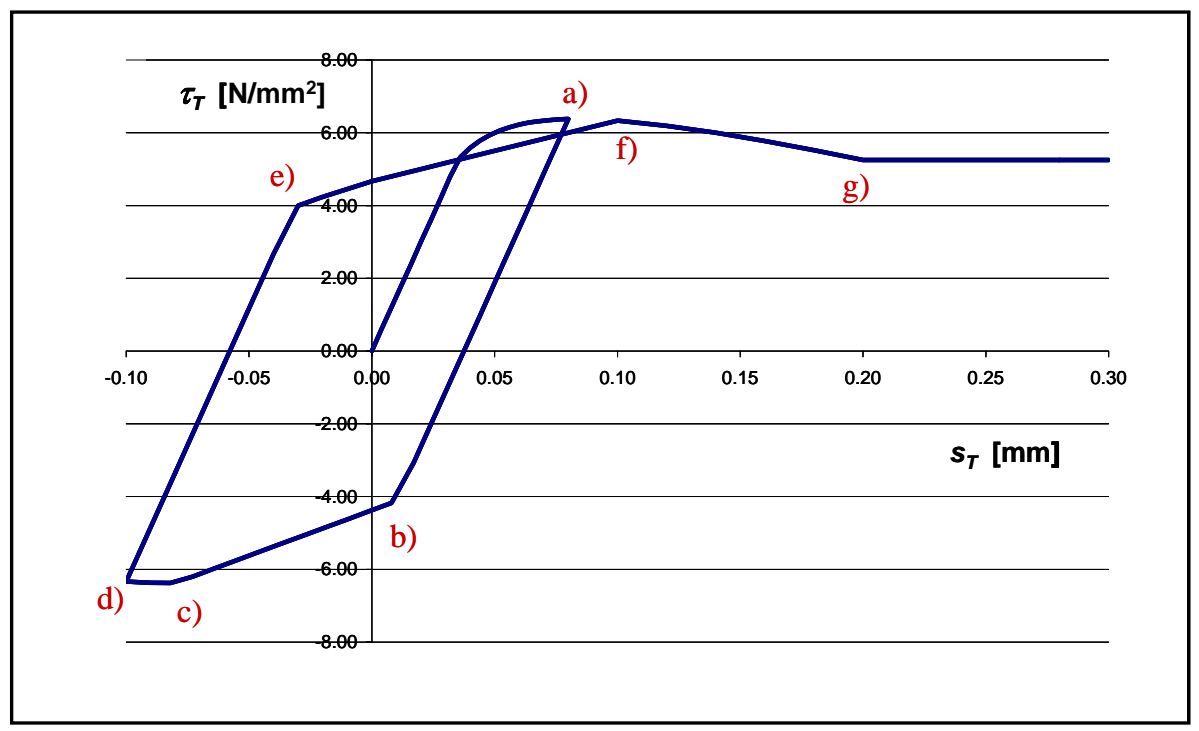

Figura 3: Curva $\tau_{T}-s_{T}$ ottenuta da una storia di carico ciclica.

L'andamento della tensione tangenziale in funzione dello spostamento relativo $S_{T}$ è riportato in Fig. 3 ed è caratterizzata dal comportamento descritti come segue.

- Dall'origine al punto (a): durante questa fase di carico il comportamento dell'interfaccia è inizialmente lineare; si manifesta in seguito un danno parziale che porta ad un comportamento non lineare e allo sviluppo dell'effetto di attrito sulla parte del RAE danneggiato.

- Dal punto (a) al punto (b): fase di scarico durante la quale non si ha evoluzione del danno. La risposta è lineare e la pendenza della curva coincide con quella del tratto iniziale della prima fase di carico.

- Dal punto (b) al punto (c): la curva cambia di pendenza perché si sviluppano spostamenti inelastici negativi senza evoluzione del danno.

- Dal punto (c) al punto (d): in tale tratto avviene un aumento del parametro di danno.

- Dal punto (d) al punto (e): durante questa fase di ricarico si osserva una pendenza del ramo coincidente con quella del tratto iniziale della prima fase di carico.

- Dal punto (e) al punto (f): la curva cambia di pendenza perché si sviluppano spostamenti inelastici senza evoluzione del danno. 
- Dal punto ( $\mathrm{f}$ ) al punto $(\mathrm{g})$ : si ha una evoluzione del parametro di danno fino a raggiungimento di decoesione completa in cui $D=1$ e la resistenza residua è dovuta solo all'effetto di attrito.

\section{Analisi di un elemento strutturale}

Si considera un semplice pannello rettangolare di dimensioni $b \times b$, vincolato tramite interfaccia al suolo rigido, come schematicamente illustrato in Fig. 4. Il pannello è caratterizzato dalle seguenti proprietà geometriche e meccaniche: $b=1000 \mathrm{~mm}, h=1000 \mathrm{~mm}$, spessore $s=1 \mathrm{~mm}$, modulo elastico $E=10000 \mathrm{MPa}$, rapporto di Poisson $v=0.25$.

Si adotta per il pannello una mesh regolare di $5 \times 5$ elementi isoparametrici a 4 nodi e si utilizzano di conseguenza 5 elementi interfaccia a 4 nodi per simulare l'effetto del contatto parete-suolo.

La storia di carico consiste nell'applicare sul lato $C D$ del pannello un carico verticale costante uniformemente distribuito $q=-1 \mathrm{~N} / \mathrm{mm}$ ed imporre sempre sul lato $C D$ uno spostamento orizzontale crescente $\eta$, il cui valore ultimo all'istante finale dell'analisi sia pari a $1 \mathrm{~mm}$.

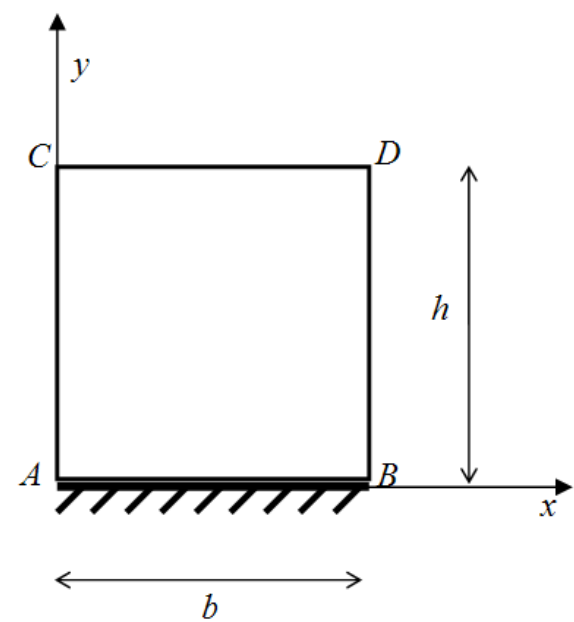

Figura 4: Pannello rettangolare vincolato tramite interfaccia ad un corpo rigido.

Si effettuano tre differenti analisi in cui si calibrano opportunamente i valori delle proprietà meccaniche dell'interfaccia al fine di verificare, per il prefissato valore di spostamento ultimo applicato nella sommità della parete, separatamente e insieme il comportamento unilatero e attritivo dell'interfaccia.

Ciascuna classe di parametri assegnati all'interfaccia costituisce un differente materiale e i rispettivi valori sono riportati in Tab. 3. In realtà, si può osservare dai dati riportati nella tabella che i tre materiali sono caratterizzati da diverse proprietà meccaniche unicamente nella direzione tangenziale.

\begin{tabular}{cccccccc}
\hline Materiale & $\begin{array}{c}\tau_{N}^{0} \\
{\left[\mathrm{~N} / \mathrm{mm}^{2}\right]}\end{array}$ & $\begin{array}{c}G_{c N} \\
{[\mathrm{~N} / \mathrm{mm}]}\end{array}$ & $\begin{array}{c}K_{N} \\
{\left[\mathrm{~N} / \mathrm{mm}^{3}\right]}\end{array}$ & $\begin{array}{c}\tau_{T}^{0} \\
{\left[\mathrm{~N} / \mathrm{mm}^{2}\right]}\end{array}$ & $\begin{array}{c}G_{c T} \\
{[\mathrm{~N} / \mathrm{mm}]}\end{array}$ & $\begin{array}{c}K_{T} \\
{\left[\mathrm{~N} / \mathrm{mm}^{3}\right]}\end{array}$ & $\mu$ \\
2 & 2 & 0.3 & 760 & 3 & 0.3 & 760 & 0.3 \\
3 & 2 & 0.3 & 760 & 1 & 0.03 & 760 & 0.3 \\
\hline
\end{tabular}

Tabella 3: Parametri del modo I e del modo II per i tre materiali di interfaccia.

La risposta meccanica del pannello viene fornita in termini di forza orizzontale totale in funzione di alcune componenti di spostamento nodali. La forza orizzontale totale $T$ è stata valutata sommando le reazioni nodali espletate dai vincoli applicati nella sommità della parete.

Nei grafici che seguono vengono mostrate le differenti risposte del pannello per i tre materiali di interfaccia considerati ed, in particolare, si riporta:

- nella Fig. 5, l'andamento di $T$ in funzione della componente orizzontale $\delta_{C}$ del punto C;

- nella Fig. 6, l'andamento di $T$ in funzione della componente verticale $\eta_{C}$ del punto $C$;

- nella Fig. 7, l'andamento di $T$ in funzione della componente di spostamento orizzontale $\delta_{B}$ del punto $B$. 


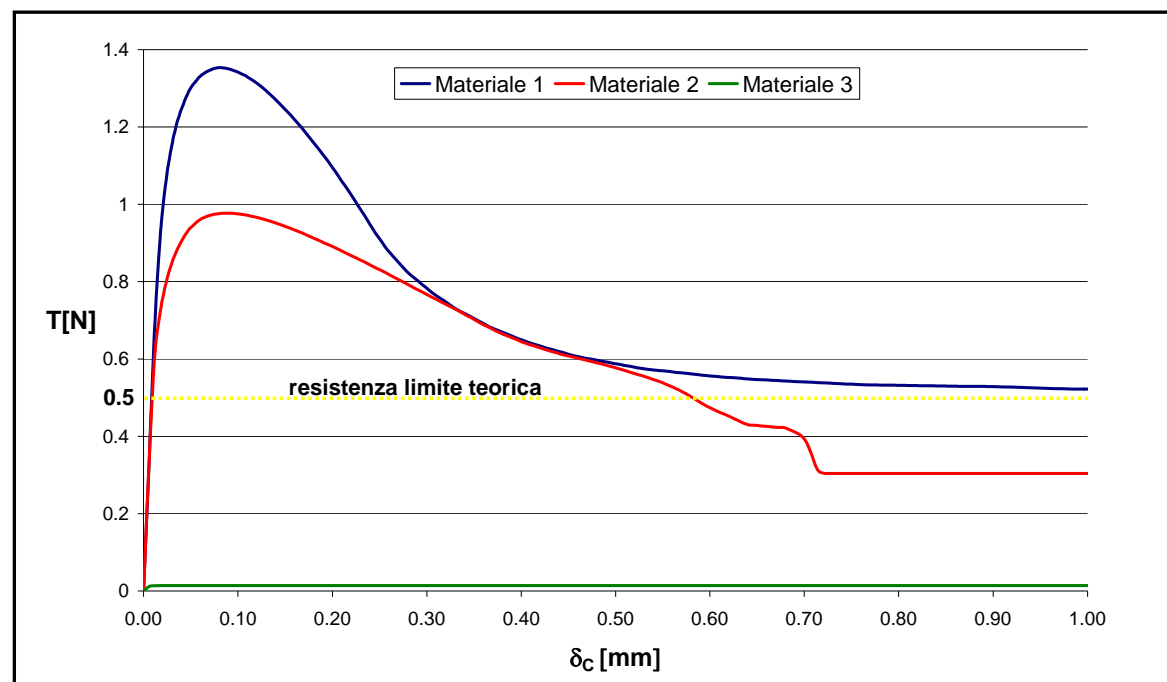

Figura 5: Forza orizzontale totale $T$ in funzione della componente di spostamento $\delta_{\mathrm{C}}$.

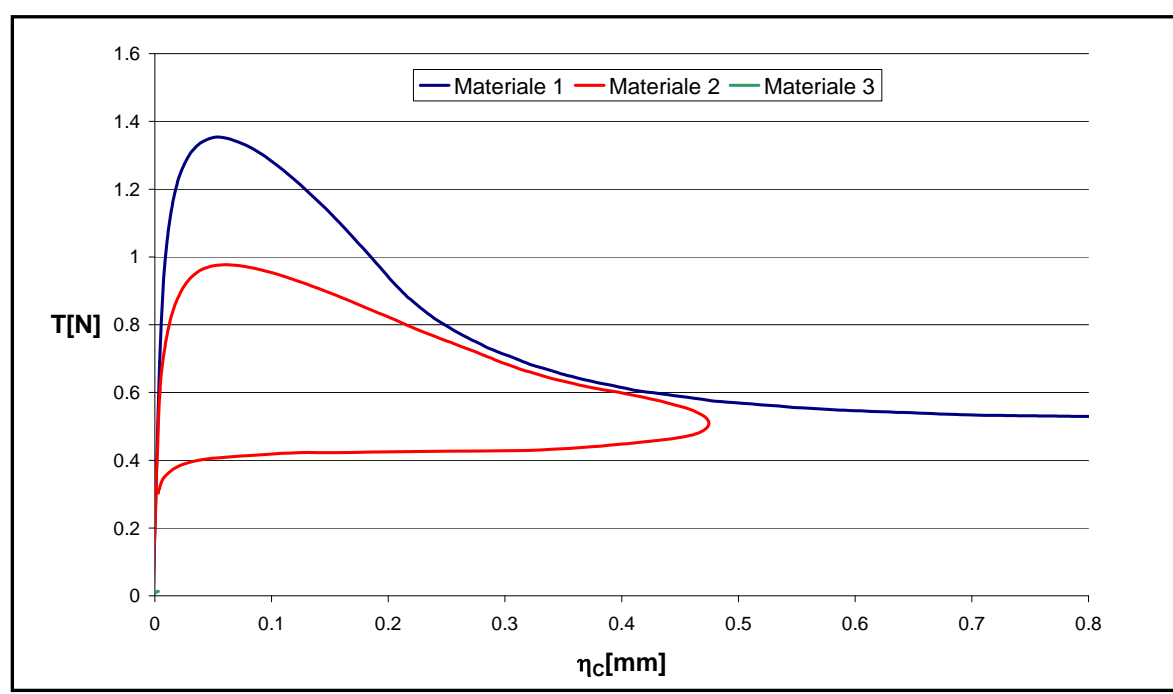

Figura 6: Forza orizzontale totale $T$ in funzione della componente di spostamento $\eta_{\mathrm{C}}$.

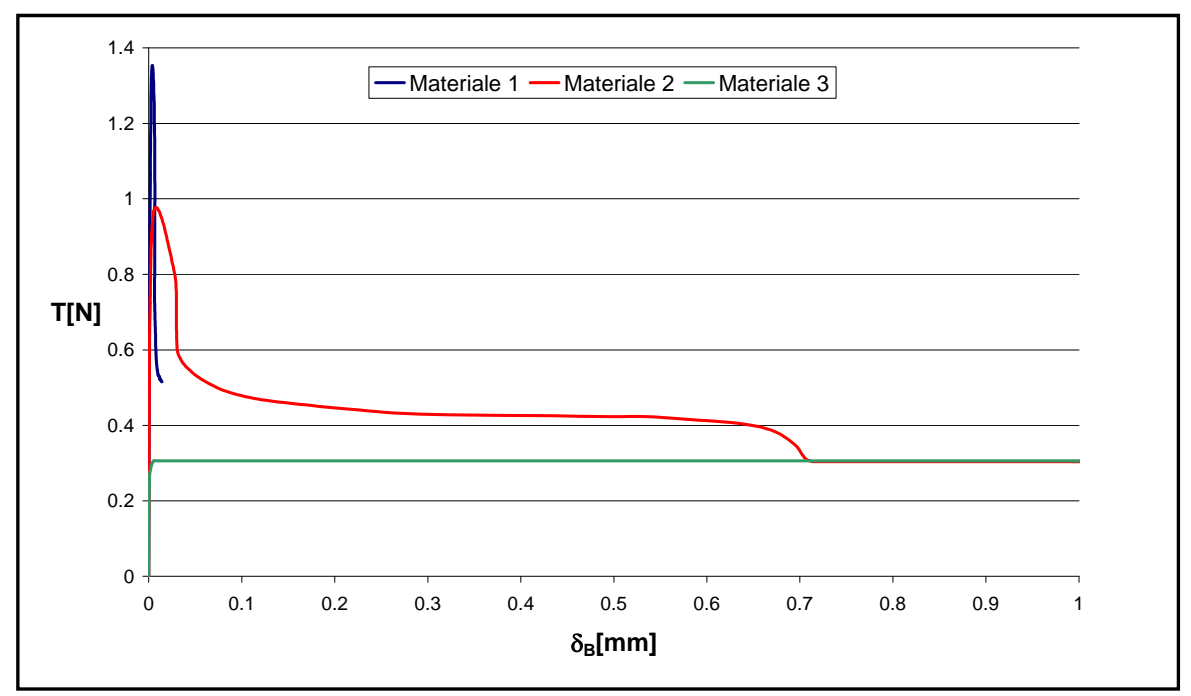

Figura 7: Forza orizzontale totale $T$ in funzione della componente di spostamento $\delta_{\mathrm{B}}$. 
Nella Fig. 8, invece, vengono mostrate per ciascun materiale di interfaccia utilizzato le configurazioni di equilibrio della parete in corrispondenza di tre valori di spostamento orizzontale del nodo $C$. In particolare viene riportata: nella Fig. 8 a il cinematismo sviluppato dal pannello con interfaccia di materiale 1, nella Fig. 8b, il cinematismo sviluppato dal pannello con interfaccia di materiale 3 e, nella Fig. 8c, il cinematismo sviluppato dal pannello con interfaccia di materiale 2.

La scelta del materiale 1 permette di verificare prevalentemente il comportamento unilatero dell'interfaccia; infatti, dalla Fig. 8a si osserva che la parete tende a sviluppare un cinematismo di ribaltamento intorno al punto $B$. Le componenti di spostamento orizzontale e verticale del punto $C$ risultano dello stesso ordine di grandezza (vedi Fig. 5 e Fig. 6) mentre la componente di spostamento orizzontale del punto $B$ è quasi assente (vedi Fig. 7).

Inoltre, nel grafico di Fig. 5 viene riportato anche il valore del carico limite ottenuto analiticamente tramite una semplice equazione di equilibrio alla rotazione del pannello intorno al polo B. Si può osservare che il valore di resistenza ultima ottenuto numericamente tende al valore di $0.5 \mathrm{~N}$ che è in pieno accordo con la previsione teorica dedotta dall'equilibrio.

Nell'applicazione effettuata con il materiale 3, si verifica sostanzialmente il comportamento attritivo dell'interfaccia. Infatti, riducendo di due ordini di grandezza i valori delle proprietà meccaniche in direzione tangenziale rispetto ai quelli normali, si favorisce un cinematismo di un puro scorrimento della parete rispetto al suolo rigido (vedi Fig. 8b). Le componenti orizzontali dei punti $B$ e $C$ crescono indefinitamente con una resistenza allo slittamento costante e pari a $0.3 \mathrm{~N}$ dovuta unicamente all'effetto dell'attrito parete-suolo (vedi Fig. 5 e Fig. 7), mentre l'andamento della componente verticale del punto $C$ risulta trascurabile (vedi Fig. 6).

Infine, è stata eseguita un'ultima analisi in cui si sono utilizzati i valori delle proprietà meccaniche relativi all'interfaccia di materiale 2. In tal caso, considerando parametri caratteristici del modo II intermedi tra quelli del materiale 1 e del materiale 3 , si è riusciti a cogliere insieme il comportamento unilatero e attritivo dell'interfaccia. Il pannello, infatti, nella fase iniziale dell'analisi tende a ruotare in senso orario intorno al polo $B$ e da un certo istante in poi inizia a ritornare nella configurazione non ruotata continuando contemporaneamente a scorrere rispetto al suolo rigido (vedi Fig. 8c).

Si può osservare dal grafico di Fig. 6 che avviene un sollevamento della parete e quindi la sua rotazione fin quando la componente verticale del punto $C$ assume valore di $0.5 \mathrm{~mm}$. La parete invece torna nella configurazione non ruotata in corrispondenza di uno spostamento orizzontale del nodo $B$ e $C$ di circa $0.7 \mathrm{~mm}$, superato tale valore si attiva il meccanismo di puro scorrimento con una resistenza allo slittamento dovuta unicamente all'effetto dell'attrito parete-suolo (vedi Fig. 5 e Fig. 7).

I risultati numerici delle applicazioni svolte hanno quindi messo in evidenza che la risposta dell'elemento finito interfaccia è perfettamente coerente al comportamento del modello di interfaccia sviluppato.

Inoltre, le elaborazioni numeriche sviluppate hanno mostrato l'affidabilità dello strumento di calcolo realizzato, il quale è stato successivamente utilizzato come mezzo di analisi per un'applicazione ingegneristica significativa.

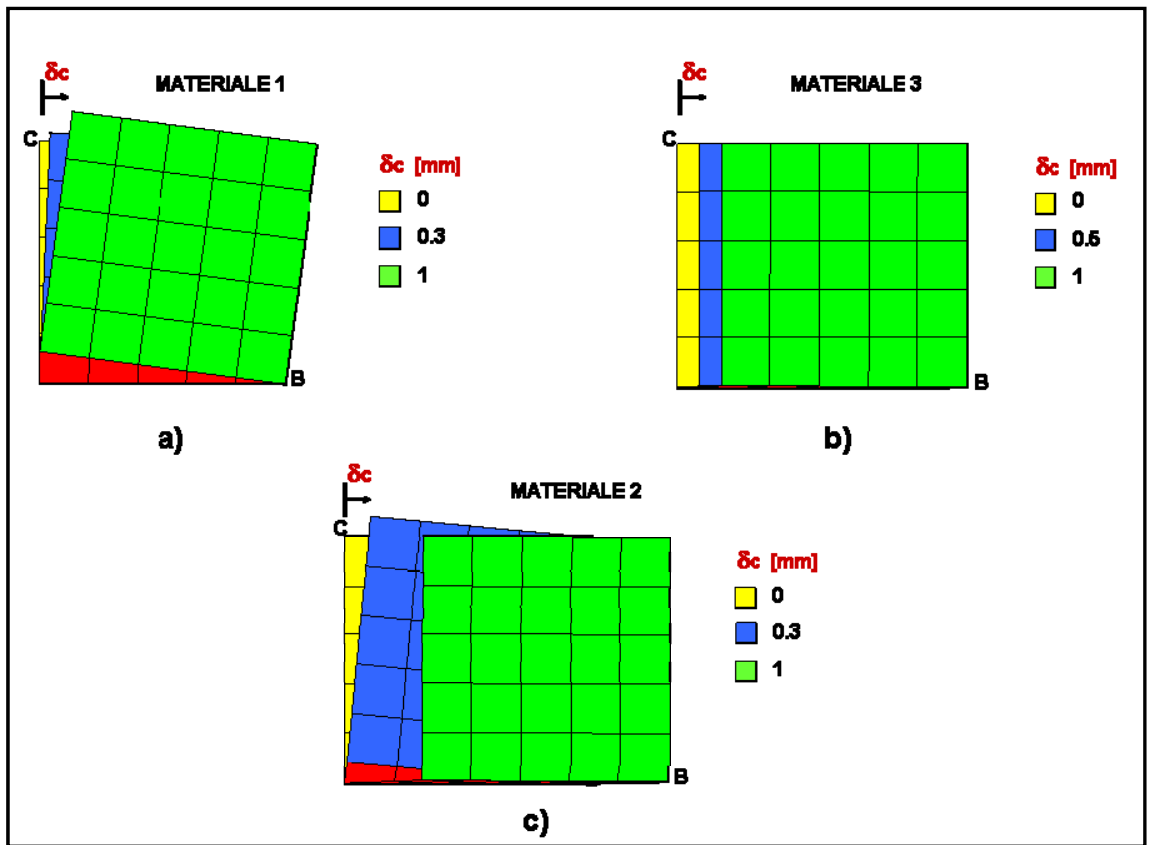

Figura 8: a) Ribaltamento (comportamento unilatero); b) Scorrimento (comportamento attritivo);

c) Ribaltamento-scorrimento (comportamento unilatero e attritivo). 


\section{Analisi di un arco in muratura}

E' stata sviluppata un'analisi numerica di un arco in muratura per il quale si è effettuata una sperimentazione presso il Laboratorio di Analisi e progettazione Strutturale, dell'Università di Cassino da parte di Cancelliere et al. [14], dove vengono riportati tutti i dati ed i risultati relativi all'attività sperimentale. Si tratta di un arco che geometricamente assume l'aspetto di un settore di corona circolare, come illustrato in Fig. 9. I dati geometrici dell'arco sono i seguenti:

- raggio esterno $R_{\text {est }}=560 \mathrm{~mm}$,

- raggio interno $R_{\text {int }}=440 \mathrm{~mm}$,

- spessore $s=250 \mathrm{~mm}$,

- anomalia di imposta $\Phi_{r}=8^{\circ}$.

L'arco è realizzato con 23 blocchi e 22 malte di allettamento. Le caratteristiche meccaniche dei materiali costituenti l'arco sono state dedotte da opportune prove sperimentali, riportate in dettaglio in 0; il modulo elastico, il rapporto di Poisson e la resistenza a compressione del mattone e della malta valgono rispettivamente:

$E_{b}=16000 \mathrm{~N} / \mathrm{mm}^{2}$

$v_{b}=0.2$

$\sigma_{y b}=38.5 \mathrm{~N} / \mathrm{mm}^{2}$

$E_{m}=1500 \mathrm{~N} / \mathrm{mm}^{2}$

$v_{m}=0.2$

$\sigma_{y m}=4.5 \mathrm{~N} / \mathrm{mm}^{2}$

Il peso per unità di volume del blocco e della malta è pari a

$\gamma_{b}=17 \mathrm{kN} / \mathrm{mm}^{3}$

$\gamma_{m}=20 \mathrm{kN} / \mathrm{mm}^{3}$.

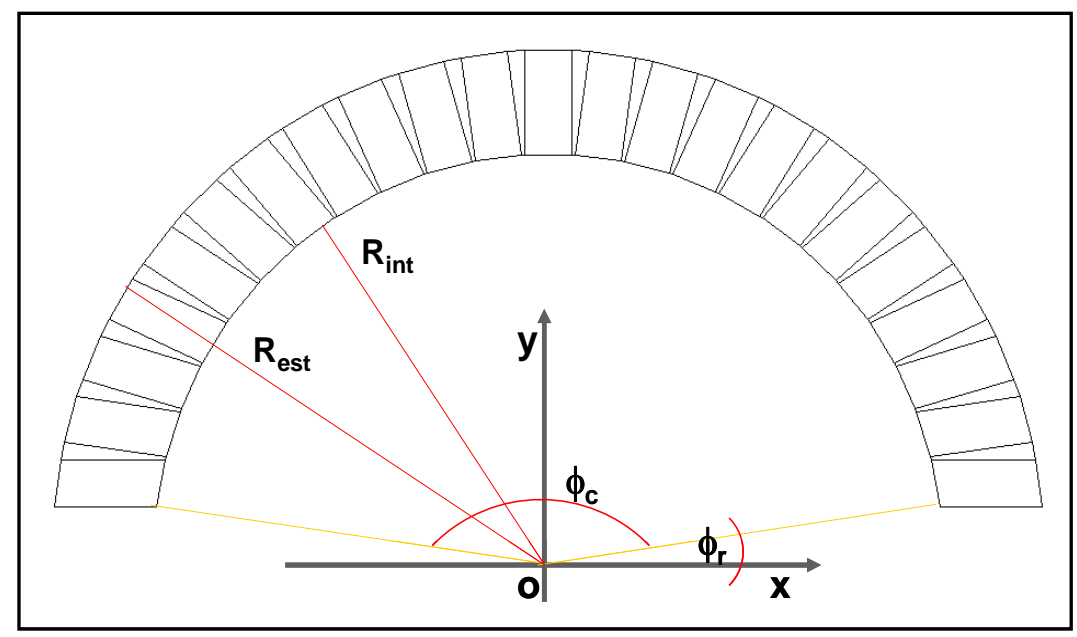

Figura 9: Modello geometrico dell'arco.

L'arco è soggetto inizialmente al peso proprio e quindi ad una forza concentrata crescente diretta verso il basso, applicata mediante l'azione espletata dal martinetto sull'estradosso dell'arco in corrispondenza del centro del $14^{\circ}$ mattone, come illustrato in Fig. 10. L'arco è vincolato alle imposte in modo da impedire scorrimenti orizzontali; in tal modo si è favorito un cinematismo di collasso caratterizzato dalla formazione delle quattro classiche cerniere, due di intradosso e due di estradosso (vedi Fig. 10). In particolare, dalla sperimentazione si è ottenuto che la prima cerniera si forma sull'estradosso tra la malta 13 ed il mattone 14, la seconda cerniera si forma sull'intradosso tra la malta 7 ed il mattone 7 , quindi si forma la cerniera sull'estradosso tra il mattone 1 e la malta 1, ed infine all'intradosso tra il mattone 19 e la malta 19 , come illustrato in dettaglio nelle fotografie riportate in Fig. 11. 


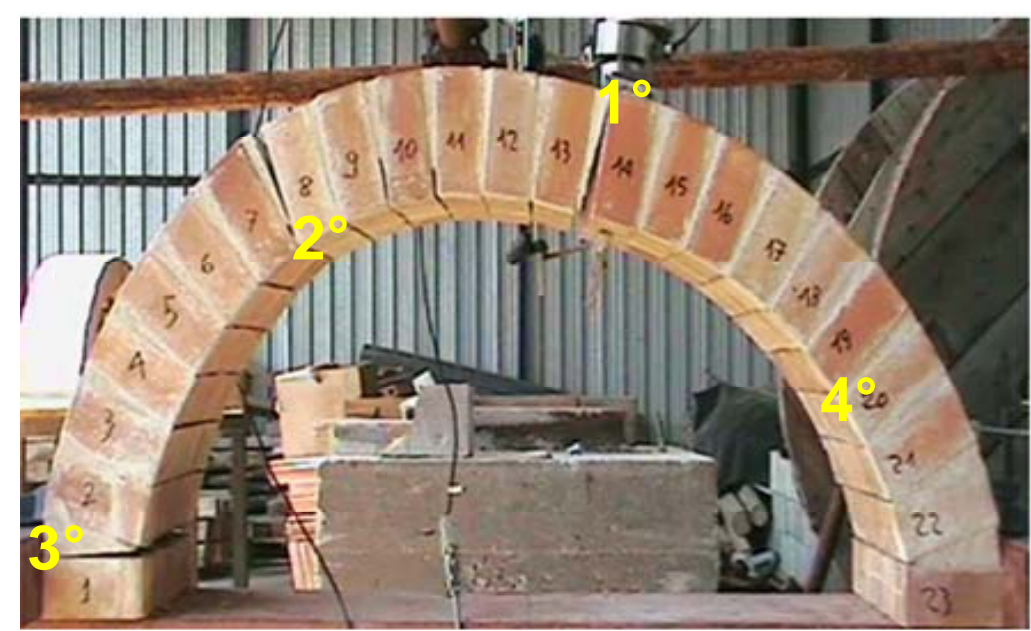

Figura 10: Arco sperimentale: numerazione dei mattoni e cinematismo di collasso.
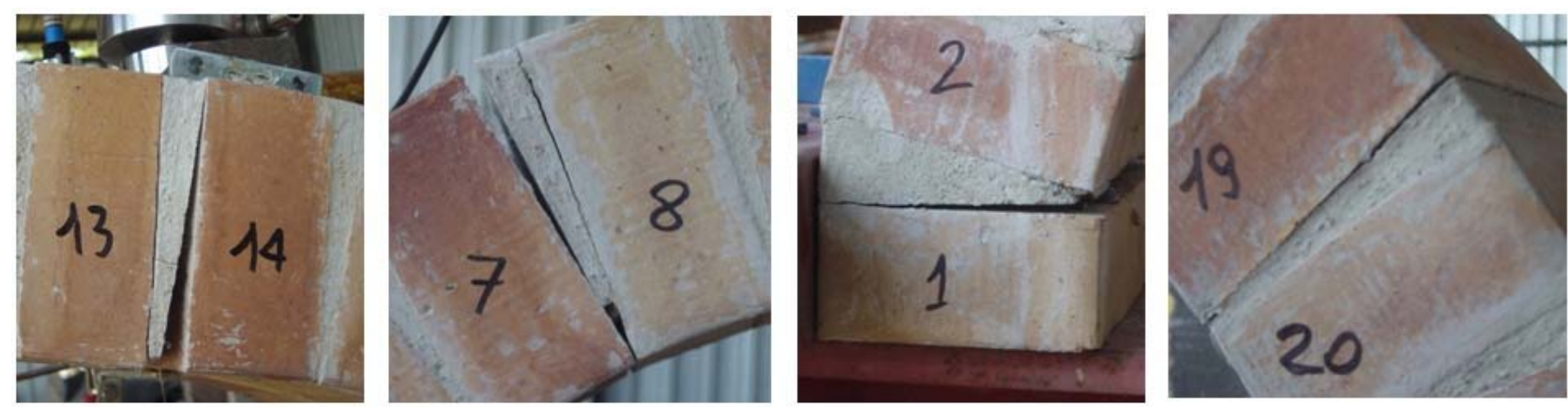

Figura 11: Immagini delle posizione delle cerniere dell'arco.

Per la modellazione dell'arco in muratura si è adottato un approccio micromeccanico, in cui i mattoni e la malta sono modellati con elementi continui mentre le interfacce blocchi-malta o mattone-base di appoggio dell'arco con superfici di discontinuità. In particolare per la malta e il mattone si sono utilizzati elementi bidimensionali elastici e il numero delle superfici di discontinuità che si sono considerate nella modellazione sono 24 e vengono simulate da elementi interfaccia a quatto nodi.

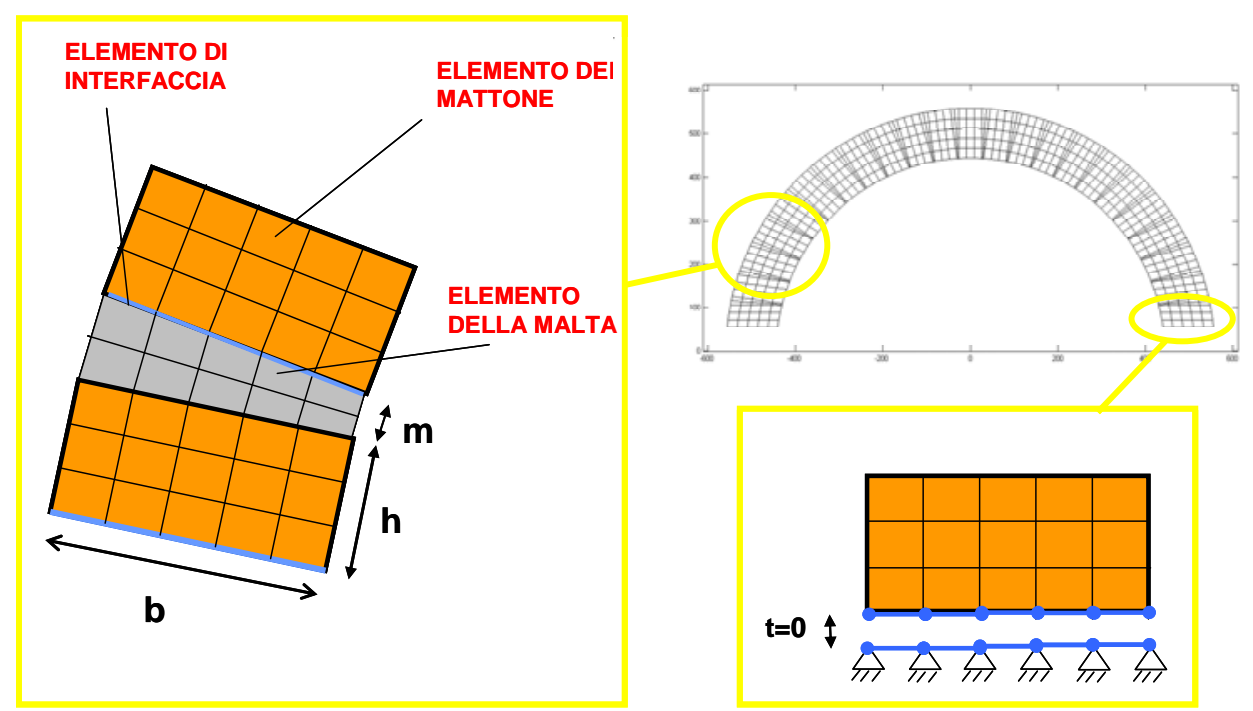

Figura 12: Discretizzazione $\left(n_{b}=3, n_{b}=5\right.$ e $\left.n_{m}=2\right)$ e vincoli. 
Nei calcoli effettuati sono state considerate diverse discretizzazioni dell'arco; i parametri utilizzati per individuare le differenti discretizzazioni sono $n_{h}, n_{b}$ e $n_{m}$ che rappresentano rispettivamente il numero di divisioni con cui viene discretizzata l'altezza $b$ del mattone, la base $b$ del mattone e lo strato di malta. In Fig. 12 è riportato un esempio della mesh nel caso in cui $n_{b}=3, n_{b}=5$ e $n_{m}=2$.

In realtà, i difetti e le irregolarità dei letti di malta, che si hanno durante le procedure di messa in opera dell'arco, hanno ridotto la sezione di contatto tra un blocco e l'altro (vedi Fig. 11). Questo importante aspetto è stato tenuto in conto nelle simulazione numeriche considerando per il parametro $b$, a seguito di un indagine statistica, non l'effettiva dimensione della base del mattone ma la sua reale lunghezza ridotta di $14 \mathrm{~mm}$.

Nelle simulazioni numeriche si è scelto di riprodurre l'azione del carico applicato sull'arco tramite martinetto, applicando una forza incrementale lungo le direzione $y$ del sistema di riferimento in corrispondenza del nodo centrale superiore della base del mattone $14^{\circ}$. Allo scopo di seguire completamente la risposta meccanica dell'arco, l'analisi numerica è stata svolta utilizzando una tecnica arc-length con controllo locale dello spostamento verticale del nodo in cui è applicato il carico. Le proprietà meccaniche degli elementi interfaccia sono state tarate andando a considerare uno strato di malta unitario.

\begin{tabular}{ccccccc}
\hline$\tau_{N}^{0}$ & $G_{c N}$ & $K_{N}$ & $\tau_{T}^{0}$ & $G_{c T}$ & $K_{T}$ & $\mu$ \\
{$\left[\mathrm{N} / \mathrm{mm}^{2}\right]$} & {$[\mathrm{N} / \mathrm{mm}]$} & {$\left[\mathrm{N} / \mathrm{mm}^{3}\right]$} & {$\left[\mathrm{N} / \mathrm{mm}^{2}\right]$} & {$[\mathrm{N} / \mathrm{mm}]$} & {$\left[\mathrm{N} / \mathrm{mm}^{3}\right]$} & \\
0.3 & 0.3 & 1500 & 3 & 0.3 & 1500 & 0.5 \\
\hline
\end{tabular}

Tabella 4: Parametri di interfaccia del modo I e del modo II.

Nelle prime tre modellazioni realizzate si sono scelti per i parametri $n_{b}$ e $n_{m}$ i medesimi valori e pari rispettivamente a 2 e 1 , mentre si è variato il parametro $n_{b}$ assumendo in ordine di esecuzione dei modelli valore 5, 10 e 20 .

I risultati delle analisi numeriche sono mostrati nel grafico di Fig. 13, in cui si riporta l'andamento della forza verticale applicata in funzione dello spostamento incrementale negativo applicato. Inoltre, sulla base del cinematismo di collasso caratterizzato dalla formazione delle quattro cerniere, il carico ultimo è stato anche valutato tramite il teorema cinematico dell'analisi limite, il quale ha fornito un valore di resistenza ultima pari a $650 \mathrm{~N}$.

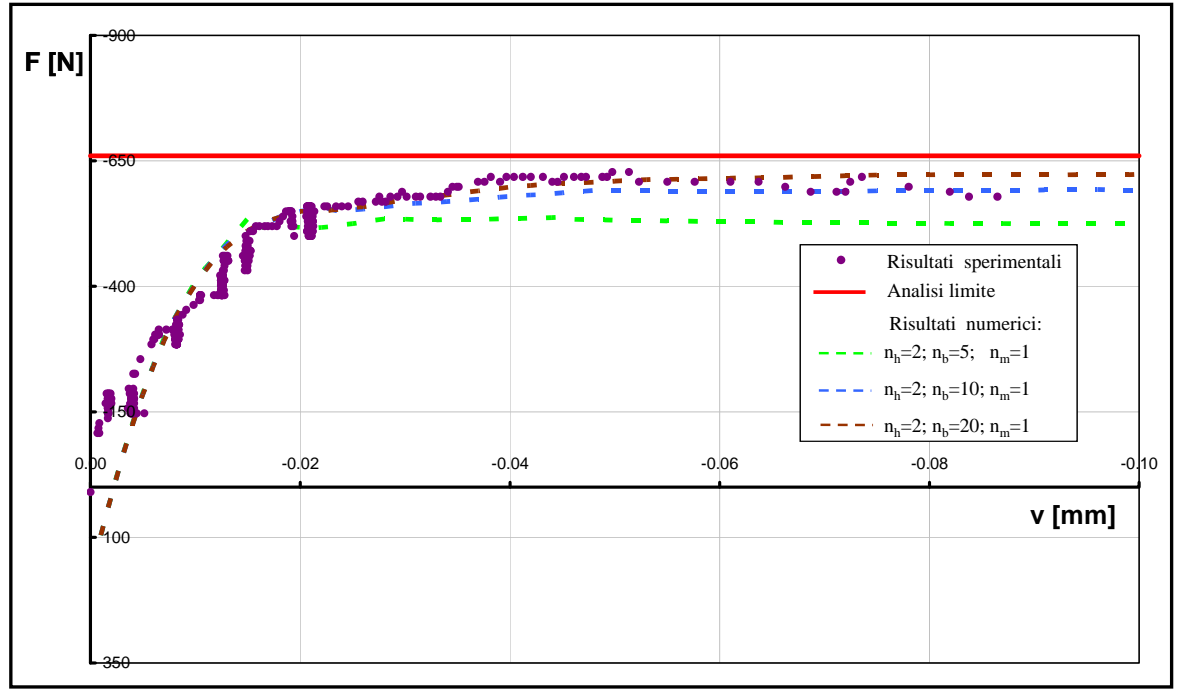

Figura 13: Risultati del primo gruppo di analisi.

Dal confronto delle analisi numeriche del primo gruppo di modellazioni insieme ai risultati sperimentali e all'analisi limite (vedi Fig.13) emerge che:

- aumentando la discretizzazione lungo la base del mattone, il risultato numerico si avvicina a quello sperimentale;

- le tre modellazioni convergono ad uno stesso risultato, poiché quest'ultimo non dipende fortemente dalla mesh adottata;

- le analisi numeriche risultano stabili;

- il carico di collasso dedotto dall'analisi limite rappresenta il limite superiore della resistenza ultima dell'arco.

Nella Fig. 14 si possono osservare, per le differenti discretizzazioni strutturali dell'arco delle prime tre modellazioni eseguite, nella sua parte sinistra, le configurazioni indeformate del solido murario mentre in quella di destra, le corrispondenti configurazioni deformate ottenute nell'istante finale dell'analisi in corrispondenza di uno spostamento 
verticale del punto di applicazione della forza pari a $0.1 \mathrm{~mm}$. Dalle deformate delle tre modellazioni, si può evidenziare che le discontinuità locali di spostamento si sono ottenute esattamente in quelle zone in cui sperimentalmente si sono manifestate le fessure.
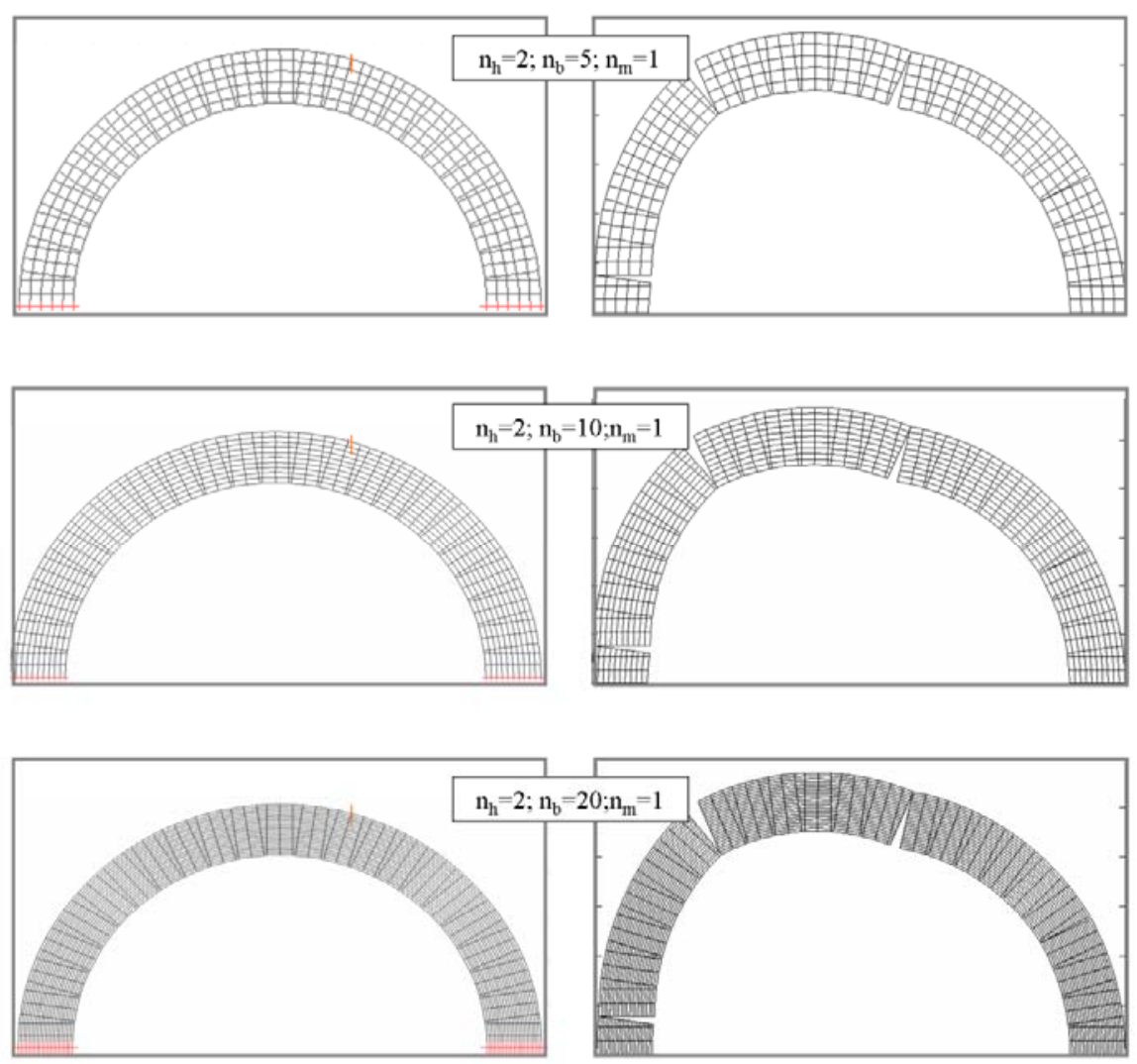

Figura 14: a) configurazione indeformata; b) configurazione deformata.

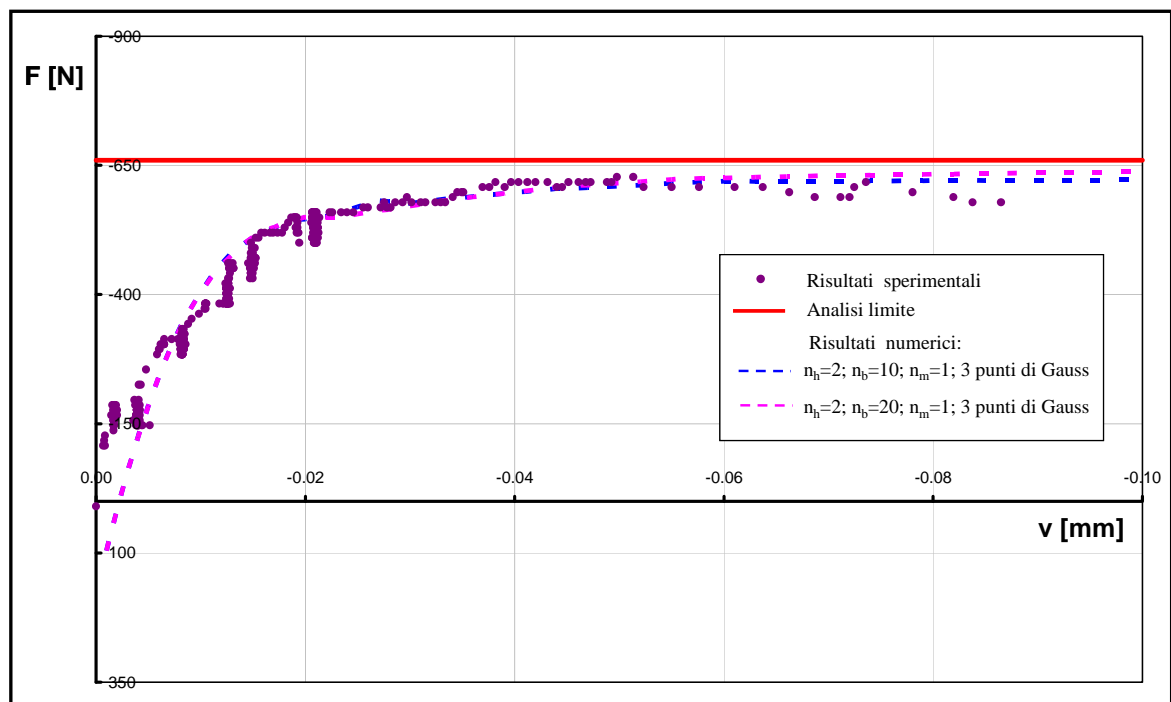

Figura 15: Risultati del secondo gruppo di analisi. 
Sono state in seguito effettuate un secondo gruppo di analisi in cui si è incrementato da 2 a 3 il numero di punti di Gauss per gli elementi interfaccia.

Dai risultati riportati in Fig. 15 emerge che:

- aumentando il numero dei punti di Gauss, le analisi convergono ad uno stesso valore del carico ultimo indipendentemente dalla mesh;

- le analisi numeriche risultano stabili.

\section{CONCLUSIONI}

L a modellazione strutturale attraverso l'utilizzo dei modelli di interfaccia risulta uno strumento efficace nello studio del comportamento delle strutture eterogenee, come quelle in muratura. Infatti in queste ultime la principale causa della risposta non lineare è dovuta generalmente a fenomeni di degrado che tendono a localizzarsi in corrispondenza delle superfici di contatto mattone-malta.

Nel presente lavoro il fenomeno di distacco giunto di malta-blocco viene simulato attraverso una versione modificata del modello costitutivo di interfaccia proposto inizialmente da Alfano e Sacco.

Le applicazioni numeriche, eseguite su semplici elementi strutturali, hanno mostrato la capacità del modello a riprodurre il comportamento non lineare dell'interfaccia giunto di malta - mattone e la robustezza dell'algoritmo implementato nel portare a termine le analisi.

Successivamente, l'applicazione riguardante l'arco murario ha dimostrato come i modelli e i metodi di calcolo proposti riescano a riprodurre il graduale sviluppo dei meccanismi di degrado che si localizzano in alcune zone del solido murario prima ancora che esso giunga a collasso.

I risultati numerici, messi a confronto con quelli sperimentali, hanno confermato una buona corrispondenza in termini quantitativi. Infatti l'andamento della curva forza - spostamento, ottenuta nel corso della sperimentazione è prossima a quella ricavata dalla simulazione.

Inoltre, grazie al particolare modello di interfaccia implementato, si è riusciti a cogliere molto bene il cinematismo di collasso sviluppato dall'arco nel corso della prova, poiché le discontinuità locali di spostamento si sono ottenute esattamente in quelle zone in cui sperimentalmente si sono manifestate le fessure.

I risultati conseguiti nel presente lavoro hanno provato che il modello di interfaccia introdotto insieme alla procedura numerica implementata hanno consentito un'analisi affidabile della risposta non lineare del solido murario, per cui in futuro il loro utilizzo potrebbe essere esteso ad ulteriori applicazioni in cui sia fondamentale non trascurare quei fenomeni di degrado che si sviluppano in certe zone critiche della muratura.

\section{RINGRAZIAMENTI}

$\mathrm{S}$ i ringrazia il Consorzio Universitario RELUIS (Dipartimento della Protezione Civile) per il finanziamento che ha reso possibile questa attività di ricerca.

\section{BIBLIOGRAFIA}

[1] D.S. Dugdale, Journal of Mechanics and Physics of Solids, 8 (1960) 100-104.

[2] G.I. Barenblatt, Advances in Applied Mechanics, 7 (1962) 55-129.

[3] H.R. Lotfi, P.B. Shing, Journal of Structural Engineering, 120(1) (1994) 63-80.

[4] G. Giambanco, L. Di Gati, Mechanics Renear\& Communications, 24(5) (1997) 503-512.

[5] L. Gambarotta, S. Lagomarsino, Earthquake Engineering And Structural Dynamics, 26 (1997).

[6] P.B. Lourenço, J. Rots, Journal of Engineering Mechanics, ASCE, 123(7) (1997) 660-668.

[7] D.V. Oliveira, P.B. Lourenço, Computers and Structures, 82 (2004) 1451-1461.

[8] G. Giambanco, Z. Mroz, Meccanica 36 (2001) 111-130.

[9] G. Alfano, E. Sacco, International Journal For Numerical Methods In Engineering; 68 (2006) 542-582.

[10] F. Fouchal, F. Lebon, I. Titeux, Construction and Building Materials, doi:10.1016/j.conbuildmat.2008.10.011.

[11] F. Ragueneau, Ch. La Borderie, J. Mazars, Mechanics Of Cohesive-Frictional Materials, 5 (2000) 607-625. 
[12] S. Marfia, G. Alfano, E. Sacco, Proceedings of the $4^{\text {th }}$ European Congress on Computational Methods in Applied Science and Engineering, ECCOMAS 2004, Jyvaskyla, Finlandia, July 24-28, 2004.

[13] G. Uva, G. Salerno, International Journal of Solids and Structures, 43 (2006) 3739-3769.

[14] I. Cancelliere, M. Imbimbo, E. Sacco, Numerical and experimental study of masonry arches. Submitted for the publication, (2008).

[15] T. Mura, Micromechanics of Defects in Solids. Second, revised edition. Martinus. (1987). 